\title{
The coughing pulsar magnetosphere
}

\author{
I. Contopoulos $\star$
}

200 Akti Themistokleous Str., Piraeus 18539, Greece

e-mail: icontop@academyofathens.gr

Received 28 March 2005 / Accepted 28 June 2005

\section{ABSTRACT}

Polar magnetospheric gaps consume a fraction of the electric potential that develops across open field lines. This effect modifies significantly the structure of the axisymmetric pulsar magnetosphere. We present numerical steady-state solutions for various values of the gap potential. We show that a charge starved magnetosphere contains significantly less electric current than one with freely available electric charges. As a result, electromagnetic neutron star braking becomes inefficient. We argue that the magnetosphere may spontaneously rearrange itself to a lower energy configuration through a dramatic release of electromagnetic field energy and magnetic flux. Our results might be relevant in understanding the recent December 27, 2004 burst observed in SGR 1806-20.

Key words. magnetohydrodynamics (MHD) - pulsars: general - stars: magnetic fields - gamma ray: bursts

\section{Introduction}

The magnetosphere of a rotating neutron star with polar magnetic field $B_{*}$, mass $M_{*} \sim M_{\odot}$, radius $r_{*} \sim 10 \mathrm{~km}$, magnetic dipole moment $\mu=r_{*}^{3} B_{*} / 2$, and angular velocity $\Omega$ is expected to radiate electromagnetic energy at a rate

$L_{\mathrm{em}} \sim \alpha \frac{B_{*}^{2} \Omega^{4} r_{*}^{6}}{c^{3}}$

$\alpha$ is a factor of order unity (Beskin 1997) ${ }^{1}$. The source of the radiation is the neutron star rotational kinetic energy which is lost at a rate

$L_{\text {kinetic }} \sim \frac{2}{5} M_{*} r_{*}^{2} \Omega \dot{\Omega}$.

It is usual to equate Eqs. (1) and (2) and thus obtain an estimate of the stellar magnetic field $B_{*}$. In general, however, the two do not have to be equal. As we shall see below, in the case of axisymmetry, electromagnetic torques need to be significantly revised.

In the context of ideal axisymmetric MHD, electric charges are available in copious amounts and move freely along magnetic field lines, shorting out any component of the electric field

^ Present address: Research Center for Astronomy \& Applied Mathematics, Academy of Athens.

${ }^{1} \alpha=\frac{1}{6} \sin ^{2} \theta$ for a misaligned dipole rotating in vacuum. In that scenario, an aligned magnetic rotator $(\theta \approx 0)$ does not radiate. However, when the neutron star is not surrounded by vacuum, one needs to consider the structure of its rotating charged relativistic Goldreich-Julian-type magnetosphere (Goldreich \& Julian 1969). In that case, the electric currents that flow through the magnetosphere lead to electromagnetic energy losses comparable to the ones for a misaligned magnetic rotator. See the Appendix for a general calculation. that might arise along the magnetic field. As a result, magnetic flux surfaces become equipotentials, and an electric field $\boldsymbol{E}$ develops across magnetic field lines $(\boldsymbol{E} \cdot \boldsymbol{B}=0)$ with magnitude

$E=\frac{r \Omega_{F}}{c} B_{\mathrm{p}}$

where, $\Omega_{F}$, a constant along magnetic flux surfaces (see below), can be thought of as the angular velocity of rotation of magnetic field lines ( $r$ is the cylindrical radius; $B_{\mathrm{p}}$ is the poloidal component of the magnetic field). The source of the electric potential across magnetic field lines is obviously the rotating magnetized conducting surface of the neutron star which acts as a unipolar inductor. For the sake of simplicity, most studies of the axisymmetric pulsar magnetosphere have assumed that the full potential drop induced across field lines along the surface of the star continues to manifest itself all along those field lines, i.e. $\Omega_{F}=\Omega$. It has been pointed out, however, (e.g. Ruderman \& Sutherland 1975) that "open magnetic field lines play a role analogous to that of conducting wires in ordinary circuits. If the wire is broken near the pulsar surface, a potential drop develops across the gap". The presence of such gaps obviously reduces the electric potential available across open field lines, and thus the electromagnetic energy power radiated at large distances. Models of particle acceleration and pair creation above the polar cap of rotation-powered pulsars yield potential drops near the surface of the star of the order of $10^{12}$ Volts (e.g. Hibschman \& Arons 2001; Arons, personal communication), and therefore, in general, $\Omega_{F}<\Omega$.

Beskin \& Malyshkin 1998 took the above well known effect into account in their calculation of the modified magnetospheric structure inside the light cylinder. In the present paper we obtain the first global solution of this problem. In Sect. 2 we 
formulate the problem and the numerical method that we implement for its solution. In Sect. 3 we obtain the structure of the magnetosphere for various values of $\Omega_{F}$ in the range $[0, \Omega]$ and argue that the magnetosphere may switch between solutions, releasing energy in the process. In Sect. 4 we discuss the relevance of our results in understanding the recent December 27, 2004 SGR-1806-20 burst. Our conclusions are summarized in Sect. 5.

\section{The differentially rotating magnetosphere}

We will work in cylindrical spatial coordinates $r, \phi, z$, and will consider only the axisymmetric case where the magnetic dipole axis is aligned with the axis of rotation. This simplification allows us to introduce the magnetic flux function $\psi(\psi / r$ is the $\phi$-component of the magnetic vector potential), the poloidal electric current function $A=A(\psi)$ (the poloidal electric current contained within the magnetic flux surface $\psi$ is equal to $A c / 2$; $\left.B_{\phi}=A / r\right)$, and the magnetic field line "rotational velocity" $\Omega_{F}=\Omega_{F}(\psi)$. The various magnetospheric physical quantities are obtained as follows:

$\boldsymbol{B}=\frac{1}{r}\left(-\psi_{z}, A, \psi_{r}\right)$,

$\boldsymbol{E}=-\frac{\Omega_{F}}{c} \nabla \psi=-\frac{\Omega_{F}}{c}\left(\psi_{r}, 0, \psi_{z}\right)$,

$\boldsymbol{J}=\frac{c}{4 \pi} \nabla \times \boldsymbol{B}=-\frac{c}{4 \pi r}\left(A^{\prime} \psi_{z}, \psi_{r r}-\frac{\psi_{r}}{r}+\psi_{z z},-A^{\prime} \psi_{r}\right)$,

$\rho_{\mathrm{e}}=\frac{1}{4 \pi} \nabla \cdot \boldsymbol{E}=-\frac{\Omega_{F}}{4 \pi c}\left(\psi_{r r}+\frac{\psi_{r}}{r}+\psi_{z z}\right)-\frac{\Omega_{F}^{\prime}}{4 \pi c}(\nabla \psi)^{2}$.

Here, and in what follows, $\psi_{x} \equiv \partial \psi / \partial x$. Also, $(\ldots)^{\prime} \equiv$ $\mathrm{d}(\ldots) / \mathrm{d} \psi$. When we neglect inertia, force balance requires that

$\frac{1}{c} \boldsymbol{J} \times \boldsymbol{B}+\rho_{\mathrm{e}} \boldsymbol{E}=0$.

Following Gruzinov (2005), we take

$c=\mu=\Omega=1$,

and thus Eq. (8) becomes

$\left(1-r^{2} \Omega_{F}^{2}\right)\left(\psi_{r r}+\frac{\psi_{r}}{r}+\psi_{z z}\right)-\frac{2 \psi_{r}}{r}=-A A^{\prime}+r^{2} \Omega_{F} \Omega_{F}^{\prime}(\nabla \psi)^{2}$.

This is a more general form of the pulsar equation than the one considered in Contopoulos, Kazanas \& Fendt (hereafter CKF) where $\Omega_{F} \equiv \Omega=1$ everywhere.

$\Omega_{F}$ is related to the magnetospheric potential drop $V_{F}$ between the axis and any magnetic flux surface $\psi$ (Eq. (5)), namely

$V_{F}(\psi)=\frac{1}{c} \int_{0}^{\psi} \Omega_{F} \mathrm{~d} \psi$

(in units $B_{*} r_{*}^{3} \Omega^{2} / 2 c^{2}$ ). This is in general different from the stellar potential drop between the pole and the footpoint on the surface of the star of the magnetic flux surface $\psi$, namely

$V_{*}(\psi)=\psi / c$.
The difference

$V(\psi) \equiv V_{*}-V_{F}=\frac{1}{c} \int_{0}^{\psi}\left(1-\Omega_{F}\right) \mathrm{d} \psi$

is just the particle acceleration gap potential which devolops along the magnetic field near the footpoint of the magnetic flux surface (e.g. Beskin 1997). In the region of closed field lines (hereafter the "dead zone"), there is no particle flow, and therefore there is no need for the formation of particle acceleration gaps. We can thus express

$\Omega_{F}(\psi)= \begin{cases}\Omega_{F o} \leq 1 & \text { along open field lines } \psi \leq \psi_{\text {open }} \\ 1 & \text { in the "dead zone" }\end{cases}$

$\Omega_{F o}(\psi)$ is determined by the particle acceleration gap microphysics and by the supply of charge carriers at the base of the magnetosphere (see discussion in Sect. 4). In our present discussion, $\Omega_{F o}(\psi)$ is essentially a free function. For the sake of simplicity, we take $\Omega_{F o}=$ const., as in Beskin \& Malyshkin (1998). The magnetospheric gap potential is, therefore, given by

$V(\psi)= \begin{cases}\left(1-\Omega_{F o}\right) \psi & \text { along open field lines } \psi \leq \psi_{\text {open }} \\ 0 & \text { in the "dead zone" }\end{cases}$

$V$ is minimal at the center of the polar cap and increases $\propto r^{2}$ as we move away from the axis.

Observational manifestation of the differential magnetospheric rotation is thought to be found in the sub-pulse slow drifts across the pulse profile in almost aligned pulsars (e.g. Rankin \& Wright 2003). Interpretation of such drifts remains still rather sketchy. We speculate that the sub-pulses are associated with the above mentioned magnetospheric gaps present around the separatrix between open and closed field lines where the need for electric charge carriers is the greatest (as we discuss below, this is where closes the electric circuit of the poloidal electric current that flows through the polar cap). In most cases with observed sub-pulse drifts $(\sim 1 \mathrm{~s}$ period pulsars) $\Omega_{F o}$ is expected to be much smaller than $\Omega$. These gaps are probably carried around the axis of rotation by the "friction" between the differentially rotating open and closed line regions, and thus their observed angular velocity is found to be close to $\Omega$.

As we mentioned before, solutions of the pulsar equation exist only for the simplest case $\Omega_{F}=1$ and $\Omega_{F}^{\prime}=0$. Even in that case, though, a strong mathematical singularity, the so called "light cylinder"

$r_{\mathrm{lc}}=1$,

makes the problem non-trivial. Note that this is just the forcefree Alfven surface, and only very recently has a numerical method been presented which allowed us to obtain a "smooth" solution that fills all space (CKF). The main features of that numerical solution (further refined in Gruzinov 2005) are:

1. The region of open field lines, the so called "polar cap", is slightly larger than the region of static dipolar field lines which cross the equator beyond the distance $r=1$, namely

$\psi_{\text {open }}=1.23$ 
(present calculation ${ }^{2}$ ). We remind the reader that $\psi_{\text {open }}=1$ for a static dipole, and therefore we see that rotation "pulls" dipolar magnetic field lines out.

2. The distribution of poloidal electric current along the open field lines is very close to the one in the Michel (1974) relativistic split monopole solution, namely

$A_{\mathrm{CKF}} \simeq$

$\begin{cases}A_{\text {Michel }} \equiv-\psi\left(2-\frac{\psi}{\psi_{\text {open }}}\right) & \text { along open field lines } \\ 0 & \text { in the "dead zone". }\end{cases}$

The electric current distribution deviates slightly from the above near $\psi \sim \psi_{\text {open }}$ where field lines follow the singular shape of the separatrix between the open and closed line regions (see Fig. 6 below).

3. The return current of the above current distribution, $A\left(\psi_{\text {open }}\right) \sim \psi_{\text {open }}$, flows along the separatrix. This implies the presence of magnetic and electric field discontinuities across the separatrix.

4. In general, the equatorial extent $r_{\mathrm{c}}$ of the "dead zone" may be taken as a free parameter (see Sect. 5, Appendix). It is very natural, however, to assume that the "dead zone" extends all the way to the light cylinder ${ }^{3}$.

5. Open field lines become monopole-like around and beyond the light cylinder.

6. $|\boldsymbol{B}|>|\boldsymbol{E}|$ everywhere ${ }^{4}$.

We are now ready to address the physically more interesting case $\Omega_{F}^{\prime} \neq 0$, in the simplest possible case where $\Omega_{F}=\Omega_{F o}=$ const. $<1$ in the open field line region, and $\Omega_{F}=1$ in the dead zone (Eq. (14)). When $\psi \leq \psi_{\text {open }}$, we can rewrite Eq. (10) in the new spatial coordinates $x \equiv \Omega_{F o} r$ and $y \equiv \Omega_{F o} z$,

$\left(1-x^{2}\right)\left(\psi_{x x}+\frac{\psi_{x}}{x}+\psi_{y y}\right)-\frac{2 \psi_{x}}{x}=-\frac{A A^{\prime}}{\Omega_{F o}^{2}}$.

Equation (19) is the same as our original equation in CKF. We thus expect that solutions of Eq. (10) will be very similar to the ones obtained in CKF. We would like to emphasize the following interesting features:

1. As in CKF, it is natural to assume that the corotating "dead zone" extends all the way to the light cylinder distance, i.e. $r_{\mathrm{c}}=r_{\mathrm{lc}}=1$. The real mathematical singularity, however,

${ }^{2}$ In CKF, with a much lower numerical resolution, we obtained a value of 1.36. Gruzinov 2005 obtained a value of 1.27 with a numerical resolution comparable to our present one.

${ }^{3}$ Gruzinov (2005) shows that this solution requires infinite magnetic fields at the point $r=1, z=0$ (in the limit of infinitesimal grid size). Uzdensky (2003) and Lyubarskii (1990) argue against infinite fields and thus conclude that the dead zone should end at some finite distance inside the light cylinder.

4 This observation counteracts criticism that the assumptions of ideal MHD may break down beyond the light cylinder (Ogura \& Kojima 2003; Spitkovsky 2004). We believe that the source of the opposite result presented in Ogura \& Kojima (2003) (their Fig. 5) is due to their numerical boundary condition, Eq. (3.3) and Fig. 1, namely that field lines become horizontal at large radial distances. is not at the light cylinder, but at a certain distance outside, the "open field light cylinder"

$r_{\text {oflc }}=\Omega_{F o}^{-1}>1$.

This is where we will apply the numerical iteration routine developed in CKF.

2. We also expect $\psi_{\text {open }} \sim 1$ as in previous solutions.

3. As in CKF, we expect to encounter similar magnetic and electric field discontinuities across the separatrix between open and closed field lines.

4. The r.h.s. of Eq. (19) is obtained through a numerical iteration along the open field light cylinder that guarantees smooth crossing of the singularity. Based on our experience, we expect this function to be very close to $-A A_{\mathrm{CKF}}^{\prime}$. Therefore, to a good approximation,

$A \simeq \Omega_{\mathrm{Fo}} A_{\mathrm{CKF}}$,

i.e. $A \propto \Omega_{F o}$. Obviously, as $\Omega_{F o} \rightarrow 0, A \rightarrow 0$. As we will see, this result has very interesting implications for the electromagnetic torques on the surface of the neutron star.

Equation (10) is elliptic with mixed boundary conditions inside and outside the open field light cylinder $r=r_{\text {offc }}$ :

1. $\psi=0$ along $r=0$, and $\psi=\psi_{\text {open }}$ along the equator beyond $r=1$ (Dirichlet boundary conditions) ${ }^{5}$.

2. $\psi_{z}=0$ (i.e. $B_{r}=0$ ) along the equator in the closed line region $r<1$ (Newman boundary condition).

3. $\psi_{r}=A A^{\prime} /\left(2 \Omega_{F o}\right)$ along the open field light cylinder $r=r_{\text {oflc }}$ (Newman boundary condition).

4. Finally, as in CKF, boundary conditions at infinity are irrelevant as long as we rescale our spatial coordinates to new ones that map the full $(r, z)=([0, \infty],[0, \infty])$ space to our finite grid size $\left(r_{\text {new }}, z_{\text {new }}\right)=([0,2],[0,1])$. Note that this is not the case for other numerical schemes where the integration is constrained within finite spatial extent (Ogura \& Kojima 2003; Goodwin et al. 2004; Gruzinov 2005).

The above show that the problem is well defined inside and outside the open field light cylinder, and therefore one can obtain solutions for a general current distribution $A=A(\psi)$. The two problems are, however, independent, and in general the solution will be discontinuous at the open field light cylinder, unless one chooses the one poloidal electric current distribution $A=A(\psi)$ that will guarantee $\psi\left(r=r_{\text {offc }}^{-}, z\right)=\psi\left(r=r_{\text {oflc }}^{+}, z\right)$. Continuity will also result in the smoothness of the solution (see above boundary condition \# 3 ). $A(\psi)$ is obtained as described in CKF by iteratively correcting to a new function

$$
\begin{gathered}
A A^{\prime}\left(\psi=\frac{1}{2}\left[\psi\left(r=r_{\text {oflc }}^{-}, z\right)+\psi\left(r=r_{\text {oflc }}^{+}, z\right)\right]\right)_{\text {new }}= \\
\frac{1}{2}\left(A A^{\prime}\left(\psi\left(r=r_{\text {oflc }}^{-}, z\right)\right)_{\text {old }}+A A^{\prime}\left(\psi\left(r=r_{\text {oflc }}^{+}, z\right)\right)_{\text {old }}\right)
\end{gathered}
$$

5 As is shown in the Appendix we are in general allowed to arbitrarily choose the equatorial extent $r_{\mathrm{c}}$ of the closed line region. In that case, $\psi_{\text {open }}$ is obtained as a solution of Eq. (10) inside the open field light cylinder, i.e. it is not an extra free parameter (see Goodwin et al. 2004 for a different point of view). 


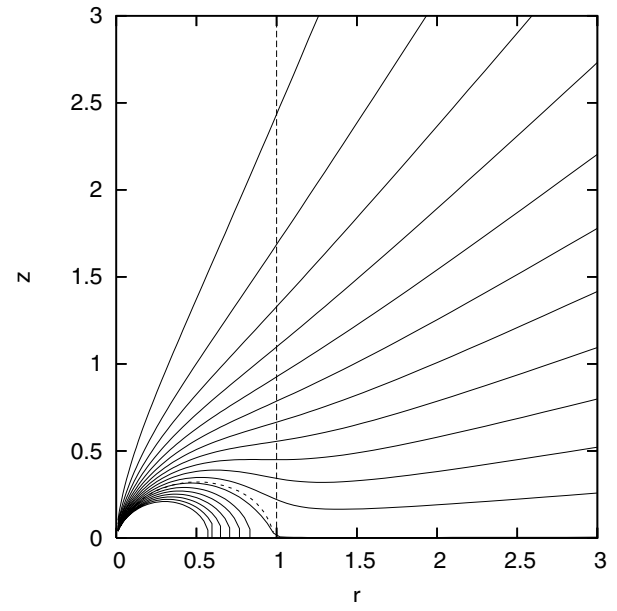

Fig. 1. $\Omega_{F}=1$ everywhere (CKF; Gruzinov 2005). Thin lines correspond to $\psi$ intervals of $0.1 . \psi=0$ along the axis. The dotted line shows the separatrix $\psi=\psi_{\text {open }}=1.23$. The mathematical singularity is at $r_{\mathrm{lc}} \equiv r_{\text {oflc }}=1$.

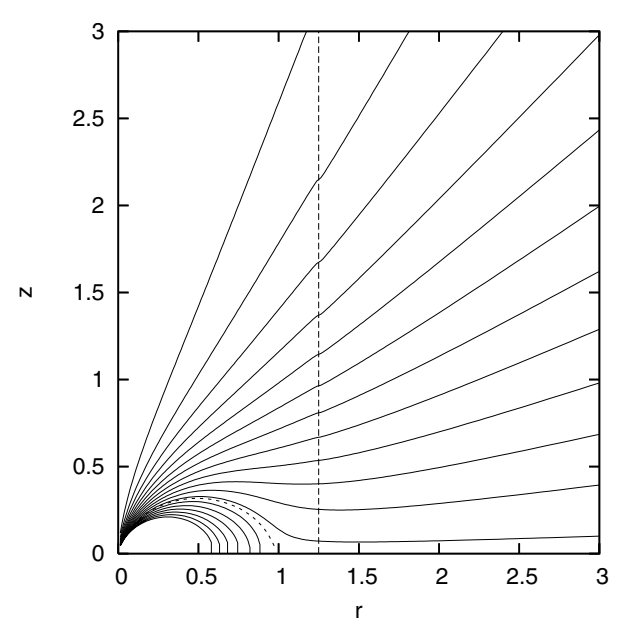

Fig. 2. $\Omega_{F o}=0.8$ in the open line region. $\psi_{\text {open }}=1.23 . r_{\text {oflc }}=1.25$.

for all grid points along the open field light cylinder. In the present work the relaxation inside each grid proceeds together with the iteration along the open field light cylinder. This improvement over the CKF method allowed for a much greater numerical resolution and a much faster speed of numerical convergence! Our numerical scheme consists of an elliptic solver with Chebyshev acceleration (Press et al. 1988) over two $100 \times 100$ numerical grids joined along the open field light cylinder. The discontinuities of $A(\psi)$ and $\Omega_{F}(\psi)$ across the separatrix between the open and closed line regions are smoothed out numerically over a distance $\delta \psi=0.05$ inside the dead zone.

\section{Steady-state magnetospheric solutions}

The various types of solutions of Eq. (10) are shown in Figs. 1-4 and B.1. Thin lines correspond to $\psi$ intervals of 0.1 . $\psi=0$ along the axis. The dotted line represents the separatrix $\psi_{\text {open. }}$. Figure 1 shows the CKF case $\Omega_{F}=1$. Here, $\psi_{\text {open }}=1.23$ within the accuracy of our numerical simulation. Figures 2 and 3 show intermediate cases with $\Omega_{F o}=0.8$ and 0.6 in the open line region respectively. $\psi_{\text {open }}=1.23$

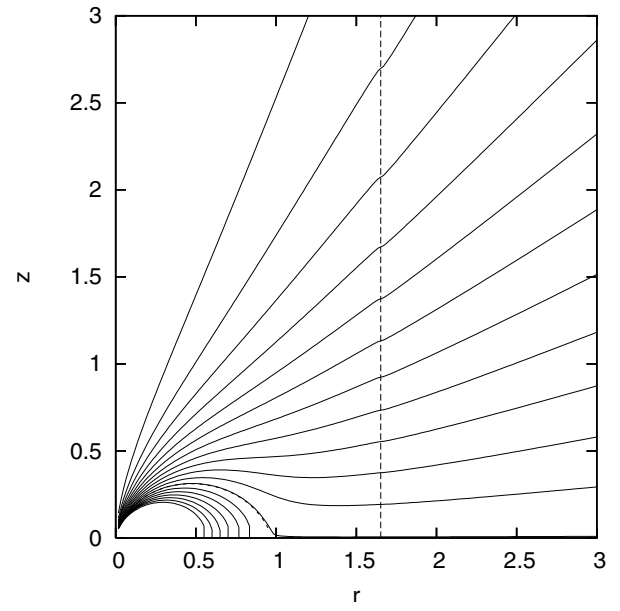

Fig. 3. $\Omega_{F o}=0.6$ in the open line region. Thin lines correspond to $\psi$ intervals of $0.1 . \psi=0$ along the axis. The dotted line shows the separa$\operatorname{trix} \psi=\psi_{\text {open }}=1.20$. The mathematical singularity is at $r_{\text {oflc }}=1.67$.

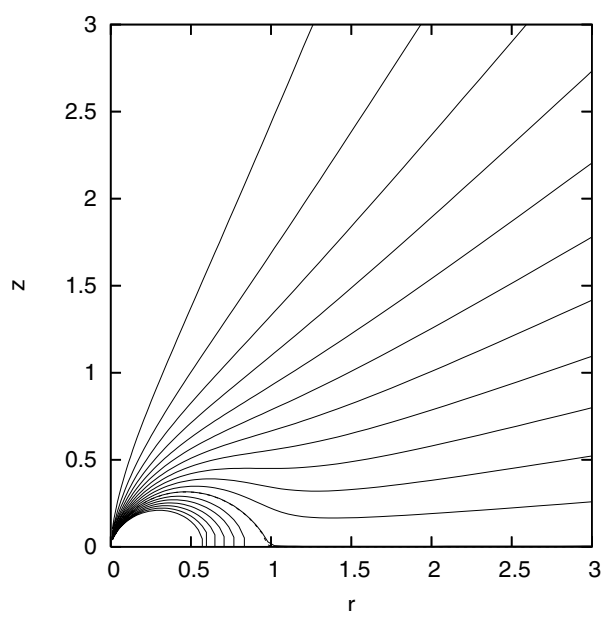

Fig. 4. $\Omega_{F o}=0$ in the open line region. $\psi_{\text {open }}=1.20 . r_{\text {offc }}=\infty$, i.e. the mathematical singularity is absent in this limiting case.

and 1.20 respectively. Figure 4 shows the limiting case with $\Omega_{F o}=0$. In that case there is no light cylinder singularity that would yield the poloidal electric current distribution $A(\psi)$. However, we showed previously that the poloidal electric current disappears, since it is obtained as a limit of solutions with $\Omega_{F o} \rightarrow 0$ in the open line region. Here, $\psi_{\text {open }}=1.20$.

The various magnetospheres show a similar poloidal magnetic field distribution. This result is understood since $B_{z}$ is approximately $\propto r^{-3}$ in the equatorial dipole-like closed line region, and therefore an approximate estimate for $\psi_{\text {open }}$ is

$\psi_{\text {open }} \sim \frac{1}{r_{\mathrm{c}}}=1$

However, they differ significantly in the amount of electric charge and electric current they contain in the open field line region, since $\rho_{\mathrm{e}} \propto \Omega_{F o}$ and $A \propto \Omega_{F o}$. As a result, they differ in the amount of electromagnetic field energy they contain in 


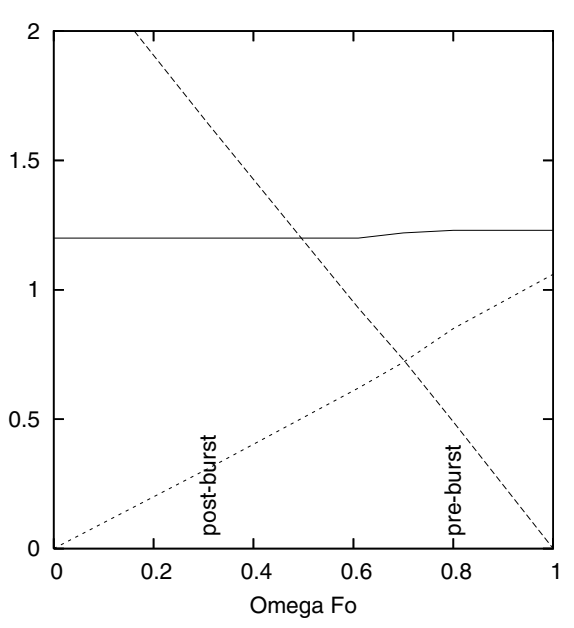

Fig. 5. Summary of our numerical solutions applied in the case of SGR 1806-20. We show here $\psi_{\text {open }}$ (continuous line), the accelerating potential $V_{\text {acc }} /\left(10^{12}\right.$ statvolt) (dashed line), and the spindown rate $|\dot{v}| / 10^{-11} \mathrm{~Hz} \mathrm{~s}^{-1}$ (short dashed line). On the plot are shown our estimates for the magnetospheric configuration before and after the December 27, 2004 burst.

the azimuthal component of the magnetic field $B_{\phi}$ and in the electric field $E$, namely

$$
\begin{aligned}
\int\left(B_{\phi}^{2}+E^{2}\right) r^{2} \mathrm{~d} r & \sim \int\left(\frac{\Omega_{F} r B_{\mathrm{p}}}{c}\right)^{2} r^{2} \mathrm{~d} r \\
& \sim \Omega_{F o}^{2} B_{*}^{2} r_{*}^{3}\left(\frac{r_{*}}{r_{\mathrm{lc}}}\right)^{3}\left(\frac{r}{r_{\mathrm{lc}}}\right) .
\end{aligned}
$$

Here, the integration distance $r$ extends to distances $\gg r_{\mathrm{lc}}$. Any evolution between the different solutions will require the release (or buildup) of the corresponding energy difference (see discussion in the next section).

We discovered that, as $\Omega_{F o}$ varies from $\Omega$ to 0 , the open field region decreases to a minimum value of about $\psi_{\text {open }} \sim 1.2$ (see Fig. 5). In the next section we will see that this numerical result might have interesting physical implications in understanding the SGR phenomenon.

Figure 6 shows the corresponding rescaled electric current distribution $A /\left(\psi_{\text {open }} \Omega_{F o}\right)$, and the rescaled distribution $A A^{\prime} /\left(\psi_{\text {open }} \Omega_{F o}^{2}\right)$, (obtained numerically) as functions of the normalized magnetic flux $\psi / \psi_{\text {open }}$. We see that indeed the electric current distributions are very similar and proportional to $\Omega_{F o}$. Let us now see how this result affects our estimation of stellar magnetic fields $B_{*}$. As we mentioned in the introduction, it is customary to estimate $B_{*}$ by equating the observed stellar spindown energy loss to the estimated electromagnetic spindown torque. As we show in the Appendix,

$$
\begin{aligned}
L_{\text {em spindown }} & =\Omega \int_{\psi=0}^{\psi_{\mathrm{open}}} A(\psi) \mathrm{d} \psi \approx \frac{2}{3} \Omega_{F o} \psi_{\mathrm{open}}^{2} \\
& \approx \frac{B_{*}^{2} \Omega^{3} \Omega_{F o} r_{*}^{6}}{4 c^{3}}\left(\frac{r_{\mathrm{lc}}}{r_{\mathrm{c}}}\right)^{2}
\end{aligned}
$$

(in real units). In general, $r_{\mathrm{c}}$ introduces one more free parameter in the problem (see Sect. 5). Let us here consider only the natural case $r_{\mathrm{c}} \sim r_{\mathrm{lc}}$ and discuss the physical significance
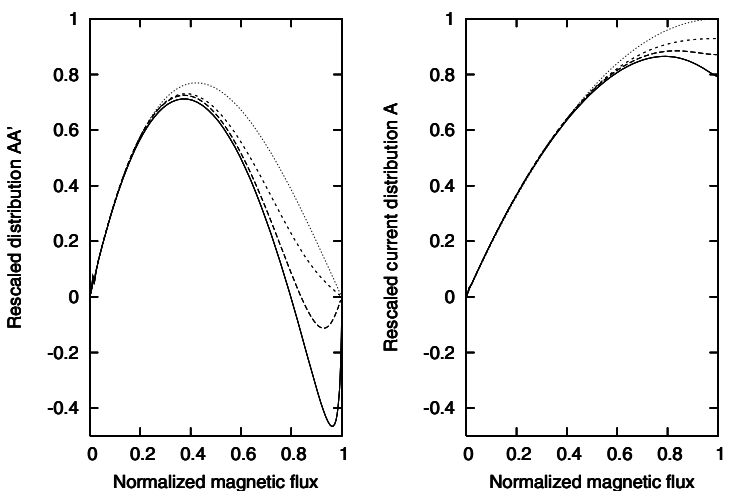

Fig. 6. The rescaled electric current distribution $A /\left(\psi_{\text {open }} \Omega_{F o}\right)$ and the rescaled distribution $A A^{\prime} /\left(\psi_{\mathrm{open}} \Omega_{F o}^{2}\right)$, as functions of the rescaled magnetic flux $\psi / \psi_{\text {open }}$ in the open line region, for $\Omega_{F o}=1,0.8$ and 0.6 (from the lower curves up respectively). The upper curves (dotted) are the ones that correspond to the Michel split monopole expression.

of $\Omega_{F o}$. Equation (25) implies that stellar magnetic field estimates need to be revised upwards over the canonical value obtained when one compares Eqs. (1) and (2). Note that when $\Omega_{F o}=0, \rho_{\mathrm{e}}=0, J=0$, i.e. no currents flow through the magnetosphere, and therefore the star will not spin down. In most cases, $\Omega_{F o} \sim[80,95] \% \Omega$ (Romani, personal communication), and therefore, the correction introduced in the stellar magnetic field estimate is in most cases practically insignificant. The correction is significant and should be taken into serious consideration for slow pulsars near the pulsar death-line, where $V_{*}\left(\psi_{\text {open }}\right) \approx 10^{12}$ Volts $=V\left(\psi_{\text {open }}\right)$ and $V_{F}\left(\psi_{\text {open }}\right) \approx 0$ (Eq. (13)).

\section{A "coughing" magnetosphere}

The solutions presented in the previous section are all steadystate solutions characterized by one parameter, $\Omega_{F o}$, which, as we argued, is determined by the particle acceleration gap microphysics. Let us imagine first that charge carriers are freely available at the base of the magnetosphere. In that case, the gap is shorted out, and the magnetosphere is described by a steadystate solution with $\Omega_{F o} \approx \Omega(\mathrm{CKF})$. Let us imagine next that the supply of charge carriers is somehow suddenly depleted. The gap will suddenly grow, and the magnetosphere will quickly evolve towards a different steady-state solution with $\Omega_{F o} \neq \Omega$. We are now going to discuss how, in our opinion, the magnetosphere may evolve from the one steady-state solution to the other. We will base our discussion on the particular example of SGR 1806-20, and its December 27, 2004 burst.

We will argue that, when the particle acceleration gap at the base of the magnetosphere suddenly grows, the magnetosphere will spontaneously evolve from a configuration with a larger open field line region and a larger poloidal electric current, to one with a smaller open field line region and a smaller poloidal electric current. One way to achieve this might be through north-south reconnection at the distance of the light cylinder. We expect a significant amount of magnetic flux $\left(\sim 5 \% \psi_{\text {open }}\right)$ to "snap" and move equatorially outward similarly to a solar coronal mass ejection (plasmoid). At the same time, the magnetosphere will release the excess energy contained in the 


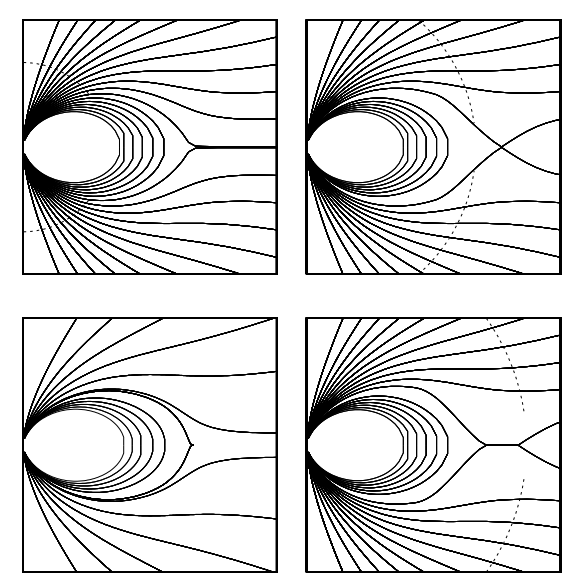

Fig. 7. Schematic magnetospheric evolution characterized as "magnetospheric coughing" (clockwise from upper left corner). In the upper left corner is shown a steady-state solution with $\Omega_{F o} \sim \Omega$. Conditions at the base of the magnetosphere changed suddenly towards a different steady-state solution with $\Omega_{F o} \neq \Omega$, and a spherical electromagnetic wave (shown with dotted line) sweeps through the open field line region at the speed of light. When the wave reaches the light cylinder region, reconnection allows the expulsion of the amount of magnetic flux required for the magnetosphere to evolve towards the new steadystate solution that corresponds to the new value of $\Omega_{F o}$ (upper right corner). The detached magnetic flux forms a "plasmoid" that escapes in the equatorial region (lower right corner). The system reaches a final steady-state shown schematically in the lower left corner, and will remain there for as long as the physical conditions that sustained the new value of $\Omega_{F o}$ at the base of the magnetosphere persist.

azimuthal component of the magnetic field $B_{\phi}$ and in the electric field $E$ through a spherical electromagnetic wave sweeping through the open field region at the speed of light ${ }^{6}$. As is shown in Eq. (24), the energy contained in that wave would grow with distance. We would like to characterize this dramatic evolution as "magnetospheric coughing" (see Fig. 7 for a schematic description).

As long as the depletion of charges persists, the magnetosphere will remain in the low $\Omega_{F o}$ state. The magnetosphere might return to a higher $\Omega_{F o}$ state where angular momentum is removed more efficiently only if charge carriers become freely available again at the base of the magnetosphere. We speculate that in such case, the magnetosphere will evolve through differential rotation between the star and the light cylinder region, and therefore the evolution will be less dramatic than the magnetospheric coughing described above.

In our example (see Fig. 5), let us choose the solution with $\Omega_{F o}=0.8$ as the pre-burst solution. Our numerical analysis yielded

$\Omega_{\text {Fo pre-burst }}=0.8$,

$\psi_{\text {open pre-burst }}=1.23$.

\footnotetext{
${ }^{6}$ In general, this will be a spherical Alfven wave moving outward at the Alfven speed.
}

Based on our detailed axisymmetric ideal MHD model, and given the observed pre-burst spindown rate $-8.5 \times 10^{-12} \mathrm{~Hz} / \mathrm{s}$, we obtain

$B_{*}=3 \times 10^{15} \mathrm{G}$,

and a corresponding accelerating potential in the magnetospheric gaps

$V_{\text {pre-burst }}=9 \times 10^{13}$ Volts.

We know that, after the burst, the spindown rate was 2.7 times smaller (Woods et al. 2005). This allows us to take

$\Omega_{\text {Fo post-burst }}=0.3$,

$\psi_{\text {open post-burst }}=1.20$.

$V_{\text {post-burst }}=3 \times 10^{14}$ Volts.

We see that both before and after the burst, the accelerating potential is of the order of $10^{14}$ Volts. Indeed, the magnetosphere is emitting pulsed radiation in both cases, only after the burst, pulsed radiation is observed to be weaker. We attribute this difference to the smaller radiation cone (due to the smaller open field line region) which might thus avoid our line of sight.

According to Eq. (24), the energy difference between the two magnetospheres is of the order of

$$
\begin{aligned}
& \left(\Omega_{F o \text { pre-burst }}^{2}-\Omega_{F o \text { post-burst }}^{2}\right) B_{*}^{2} r_{*}^{3}\left(\frac{r_{*}}{r_{\mathrm{lc}}}\right)^{3}\left(\frac{r}{r_{\mathrm{lc}}}\right) \sim \\
& 10^{47}\left(\frac{r_{*}}{r_{\mathrm{lc}}}\right)^{3}\left(\frac{r}{r_{\mathrm{lc}}}\right) \text { erg. }
\end{aligned}
$$

According to 33, the energy contained in the spherical blast wave will be comparable to the apparent burst luminosities observed on earth (e.g. Yamazaki et al. 2005) at distances $r / r_{\mathrm{lc}} \sim\left(r_{\mathrm{lc}} / r_{*}\right)^{3}$. We would like to defer a more detailed discussion of the burst energetics to a future work.

\section{Conclusions}

In our present work we presented global numerical solutions of the generalized pulsar equation that describe the steadystate structure of axisymmetric rotating neutron star magnetospheres. We have introduced two new parameters besides the neutron star angular velocity $\Omega$,

- $\Omega_{F o}$, the angular velocity of rotation of open field lines. This quantity is related to the particle acceleration gaps at the base of the magnetosphere (the closer $\Omega_{F o}$ is to $\Omega$, the smaller the gap), and is determined by gap microphysics outside the context of our present ideal MHD formulation.

- $r_{\mathrm{c}}$, the maximum equatorial extent of the closed line region (see Appendix). We speculate that $r_{\mathrm{c}}$ might be determined by inertial effects outside the context of our present ideal MHD formulation. 
Note that, in our global solutions, $\psi_{\text {open }}$ (the amount of open field lines) is determined self-consistently, and consequently it is not a free parameter (see, however, Goodwin et al. 2004, for a different point of view). Similarly to CKF, the poloidal electric current distribution that guarantees smoothness and continuity at the open field light cylinder is obtained iteratively, and an approximate analytic expression is given. Our results generalize the solution presented in CKF; Gruzinov (2005).

We also obtained a generalized expression for the steadystate spindown magnetospheric energy losses (Eq. (25)), which is different from the canonical one for a misalingned magnetic rotator. Magnetospheres with different values of $\Omega_{F o}$ and/or $r_{\mathrm{c}}$ contain different amounts of electric currents, and therefore spin down differently. This changes slightly our estimates of stellar magnetic fields $B_{*}$ (see also Harding et al. 1999, for a relevant discussion in the case of magnetar magnetic field estimates). More importantly, however, this might have serious implication in the calculation of the magnetic braking index $n \equiv \Omega \ddot{\Omega} / \dot{\Omega}^{2}$. One can easily check (Eq. (25)) that any functional dependence of $\Omega_{F}$ and $\psi_{\text {open }}$ different from the canonical one $\Omega_{F} \propto \Omega$, and $\psi_{\text {open }} \propto \Omega$ will yield a braking index $n \neq 3$ as obtained observationally (Contopoulos \& Spitkovsky, in preparation).

Finally, we argued that the magnetosphere may spontaneously evolve between steady-state configurations characterized by different values of $\Omega_{F o}$ and/or $r_{\mathrm{c}}$. The evolution from a high to low value of $\Omega_{F o}$ and/or low to high value of $r_{\mathrm{c}}$ will result in the dramatic release of a significant amount of electromagnetic field energy and magnetic flux. The return to the former configuration will be less dramatic, since it will require the buildup of the corresponding electromagnetic field energy difference. Our results might be relevant in understanding the SGR burst phenomenon.

Acknowledgements. We would like to thank Christos Eftymiopoulos and Demos Kazanas for their support in reviving this intriguing problem. We would also like to thank Jonathan Arons, Roger Blandford, Roger Romani, and Anatoly Spitkovsky for interesting discussions and comments. We would finally like to acknowledge the contribution of the unknown referee in improving the presentation of our ideas.

\section{Appendix A: Pulsar spindown estimates}

When a neutron star is not surrounded by vacuum, the rotating charged relativistic Goldreich-Julian-type magnetosphere is threaded by poloidal and toroidal electric currents. We will consider only the axisymmetric case for simplicity. Two large scale poloidal electric current circuits (north \& south) are generated. These flow only along open field lines, and close along the surface of the star at the two polar caps where they generate electromagnetic torques antiparallel to the angular momentum of the neutron star

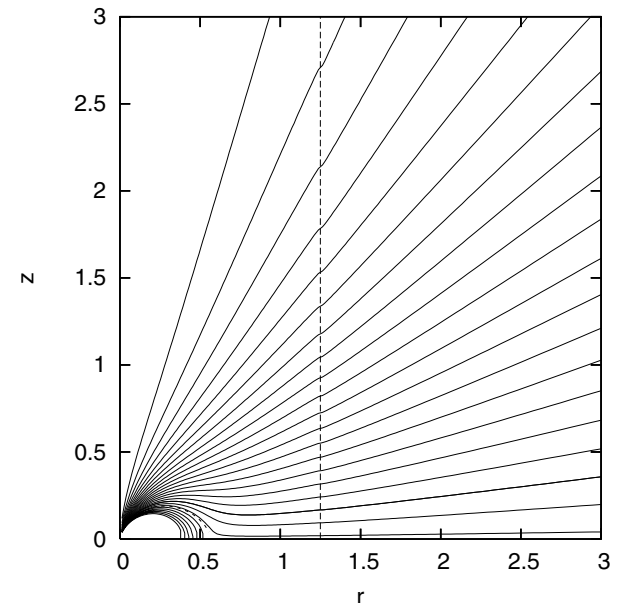

Fig. B.1. A solution with 2.7 times more efficient spindown than the solution shown in Fig. 2. The "dead zone" extends up to $r_{\mathrm{c}}=0.59$. $\Omega_{F o}=0.8 . \psi_{\text {open }}=2.03$.

through any stellar cross section $\mathrm{d} S$ threaded by poloidal electric current density $J$. One can easily check that the stellar kinetic energy loss through the above torques is given by

$L_{\text {em spindown }}=\Omega \int_{\psi=0}^{\psi_{\text {open }}} A(\psi) \mathrm{d} \psi \simeq \frac{2}{3} \Omega \Omega_{F o} \psi_{\text {open }}^{2} \approx \Omega_{F o}$

(our expression accounts for the two hemispheres, north \& south). We made use of the numerical result $\psi_{\text {open }} \approx 1.23$. At the same time, the magnetosphere radiates electromagnetic energy

$\frac{c}{4 \pi} r E_{p} B_{\phi} \mathrm{d} S$

through any cross section $\mathrm{d} S$ in the region of open field lines. One can easily check that the total electromagnetic energy loss through the above Poynting flux is given by

$L_{\mathrm{em}}=\int_{\psi=0}^{\psi_{\mathrm{open}}} A(\psi) \Omega_{F}(\psi) \mathrm{d} \psi \simeq \frac{2}{3} \Omega_{F o}^{2} \psi_{\text {open }}^{2} \approx \Omega_{F o}^{2}$.

$\Omega_{F}$ is in general smaller than $\Omega$, and therefore, $L_{\mathrm{em}}$ is in general less than $L_{\mathrm{em}}$ spindown. The difference between the two is consumed in the particle acceleration gaps that develop along open field lines, namely

$$
\begin{aligned}
L_{\text {particles }} & =L_{\text {em spindown }}-L_{\mathrm{em}}=\int_{\psi=0}^{\psi_{\mathrm{open}}} A(\psi)\left(\Omega-\Omega_{F}(\psi)\right) \mathrm{d} \psi \\
& \simeq \frac{2}{3} \Omega_{F o}\left(1-\Omega_{F o}\right) \psi_{\mathrm{open}}^{2} \approx \Omega_{F o}\left(1-\Omega_{F o}\right) .
\end{aligned}
$$

The above expressions are normalized to the Goldreich-Julian value

$L_{\mathrm{GJ}} \equiv \frac{B_{*}^{2} \Omega^{4} r_{*}^{6}}{4 c^{3}}$

\section{Appendix B: Alternative magnetospheric solutions}

In solving Eq. (10), we have all along argued that nature will choose the most natural solution, namely the one with the maximum extent of the "dead zone". A competing to the above scenario might be one where the extent of the "dead zone" is a 
free parameter $r_{\mathrm{c}}$ (Goodwin et al. 2004). Since $\Omega_{F}$ introduces one more free parameter in the problem, we will consider only one representative case with $\Omega_{F o}=0.8$. Equation (10) can be solved numerically as described before. In this scenario, solutions with a smaller "dead zone" are also more efficient in removing angular momentum from the spinning star (Eq. (A.2)). As an example, we take $r_{\mathrm{c}} \sim 0.59$ and obtain $\psi_{\text {open }}=2.03$ (Fig. B.1). This solution may evolve rapidly through reconnection towards the solution shown in Fig. 2 with $r_{\mathrm{c}} \sim 1$, $\psi_{\text {open }}=1.23$, and thus yield a spindown rate 2.7 times lower, releasing at the same time a significant amount of magnetic field energy. Note that the system is an efficient radiator through particle acceleration processes both before and after the burst $\left(\Omega_{F o}<1\right)$.

\section{References}

Beskin, V. S. 1997, Physics-Uspekhi, 40, 659

Beskin, V. S., \& Malyshkin, L. M. 1998, MNRAS, 298, 847

Contopoulos, I., Kazanas, D., \& Fendt, C. 1999, ApJ, 511, 351
Goldreich, P., \& Julian, W. H. 1969, ApJ, 157, 869

Goodwin, S. P., Mestel, J., Mestel, L., \& Wright, G. A. E. 2004, MNRAS, 349, 213

Gruzinov, A. 2005, Phys. Rev. Lett., 94

Harding, A. K., Contopoulos, I., \& Kazanas, D. 1999, ApJ, 525, 125

Hibschman, J. A., \& Arons, J. 2001, ApJ, 554, 624

Lyubarskii, Y. E. 1990, Sov. Astron. Lett., 16, 16

Michel, F. C. 1974, ApJ, 192, 713

Ogura, J., \& Kojima, Y. 2003, Progr. Theor. Phys., 109, 619

Palmer, D. M., Barthelmy, S., Gehrels, N., et al. 2005 [arXiv: astro-ph/0503030]

Press, W. H., Flamery, B. P., Teukolsky, S. A., \& Vetterling, W. T. 1988, Numerical Recipes (Cambridge: Cambridge Univ. Press)

Rankin, J. M., \& Wright, G. A. E. 2003, A\&ARv, 12, 43

Ruderman, M. A., \& Sutherland, P. G. 1975, ApJ, 196, 51

Spitkovsky, A. 2004, IAUS, 218, 357

Uzdensky, D. A. 2003, ApJ, 598, 446

Woods, P. M., Kouveliotou, C., Gogus, E., et al. 2005, Astronomer's Telegram, No. 407

Yamazaki, R., Ioka, K., Takahara, F., \& Shibazaki, N. 2005 [arXiv: astro-ph/0502320] 